\title{
A novel genomic panel as an adjunctive diagnostic tool for the characterization and profiling of breast Fibroepithelial lesions
}

Yirong Sim ${ }^{1,2^{*}}$ (D), Gwendolene Xin Pei $\mathrm{Ng}^{1,3,4}$, Cedric Chuan Young Ng ${ }^{3,4}$, Vikneswari Rajasegaran ${ }^{3,4}$, Suet Far Wong ${ }^{3,4}$,

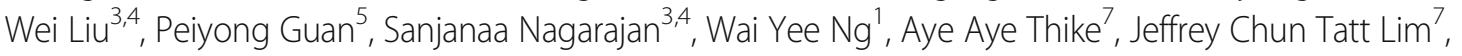
Nur Diyana Binte Md Nasir ${ }^{7}$, Veronique Kiak Mien Tan ${ }^{1,2}$, Preetha Madhukumar ${ }^{1,2}$, Wei Sean Yong ${ }^{1,2}$, Chow Yin Wong ${ }^{2}$, Benita Kiat Tee Tan ${ }^{1,2}$, Kong Wee Ong ${ }^{1,2}$, Bin Tean Teh ${ }^{4,6}$ and Puay Hoon Tan ${ }^{7,8}$

\begin{abstract}
Background: Known collectively as breast fibroepithelial lesions (FELS), the common fibroadenomas (FAs) and the rarer phyllodes tumors (PTs) are a heterogenous group of biphasic neoplasms. Owing to limited tissue availability, inter-observer variability, overlapping histological features and heterogeneity of these lesions, diagnosing them accurately on core biopsies is challenging. As the choice management option depends on the histological diagnosis; a novel 16-gene panel assay was developed to improve the accuracy of preoperative diagnosis on core biopsy specimens.

Methods: Using this 16-gene panel, targeted amplicon-based sequencing was performed on 275 formalin-fixed, paraffin-embedded (FFPE) breast FEL specimens, archived at the Singapore General Hospital, from 2008 to 2012.

Results: In total, 167 FAs, 24 benign, 14 borderline and 6 malignant PTs, were profiled. Compared to FAs, PTs had significantly higher mutation rates in the TERT promoter $(p<0.001)$, RARA $(p<0.001), F L N A, R B 1$ and TP53 $(p=0.002$, 0.020 and 0.018 , respectively). In addition to a higher mutational count $(p<0.001)$, TERT promoter $(p<0.001)$, frameshift, nonsense and splice site ( $p=0.001,<0.001$ and 0.043 , respectively) mutations were also frequently observed in PTs.

A multivariate logistic regression model was built using these as variables and a predictive scoring system was developed. It classifies a FEL at low or high risk (score $<1$ and $\geq 1$, respectively) of being a PT. This scoring system has good discrimination ( $\mathrm{ROC}$ area $=0.773,95 \% \mathrm{Cl}: 0.70$ to 0.85 ), calibration $(p=0.945)$ and is significant in predicting PTs $(p<0.001)$.
\end{abstract}

Conclusion: This novel study demonstrates the ability to extract DNA of sufficient quality and quantity for targeted sequencing from FFPE breast core biopsy specimens, along with their successful characterization and profiling using our customized 16-gene panel. Prospective work includes validating the utility of this promising 16-gene panel assay as an adjunctive diagnostic tool in clinical practice.

Keywords: Breast, Fibroepithelial lesion, Fibroadenoma, Phyllodes, Genomic test, Core biopsy

\footnotetext{
* Correspondence: sim.yirong@singhealth.com.sg;

Sim.yirong@singhealth.com.sg

${ }^{1}$ Division of Surgical Oncology, National Cancer Centre Singapore, Singapore,

Singapore

${ }^{2}$ SingHealth Duke-NUS Breast Centre, Singapore General Hospital, Singapore,

Singapore

Full list of author information is available at the end of the article
}

(c) The Author(s). 2019 Open Access This article is distributed under the terms of the Creative Commons Attribution 4.0 International License (http://creativecommons.org/licenses/by/4.0/), which permits unrestricted use, distribution, and reproduction in any medium, provided you give appropriate credit to the original author(s) and the source, provide a link to the Creative Commons license, and indicate if changes were made. The Creative Commons Public Domain Dedication waiver (http://creativecommons.org/publicdomain/zero/1.0/) applies to the data made available in this article, unless otherwise stated. 


\section{Background}

Breast fibroepithelial lesions (FELs) belong to a family of biphasic neoplasms, characterized by the proliferation of both epithelial and stromal components. The common benign fibroadenomas (FAs) are distinguished from the much rarer phyllodes tumors (PTs) by the presence of leaf-like stromal fronds and increased stromal cellularity in the latter. Phyllodes tumors, which comprise less than $1 \%$ of all breast tumors, can be classified into benign $(\mathrm{BEN})$, borderline (BDR) and malignant (MAL) grades based on the assessment of five histological parameters-stromal cellularity and overgrowth, nuclear atypia, cellular pleomorphism, mitotic activity and tumor borders $[1,2]$. Compared to western counterparts, a higher incidence rate of PTs is observed in women of Asian descent [2-4].

Core needle biopsies are recommended for preoperative histological diagnoses of breast lesions as this technique is minimally invasive, cost-effective and can be carried out in an outpatient setting $[5,6]$. In the context of breast FELs, the choice therapeutic option hinges on the histological diagnosis-either observation or enucleation for benign FAs, to a surgical resection with wide margins (wide excision) for PTs [6-8]. However, discriminating between the two entities preoperatively, in particular cellular FELs, can be challenging, especially with limited available material from core biopsies, due to overlapping histological features and lesional heterogeneity, compounded by inter-observer variability [8-11]. Thus, there is a need for a highly specific and sensitive pre-operative histopathologic diagnosis based on the limited material from core biopsies to avoid over- or under-treatment and to reduce unwarranted anxiety and cost to the patient [12]. Several studies have explored the genomic landscapes of breast FELs. MED12 is the only gene that has mutations occurring frequently in FAs and PTs of all grades [13, 14], suggesting a biological link between these FELs that share morphological features and genetic abnormalities [15]. Other mutations observed more commonly in PTs consist of RARA, FLNA, SETD2, KMT2D and the TERT promoter gene abnormalities [16]. The frequency of mutations in the TERT promoter was observed to increase with increasing grade of PTs, implying its possible role in driving the progression of PTs and differentiating FAs from the PTs $[17,18]$. Other oncogenes associated with the borderline and malignant spectrum of PTs include TP53, RB1, EGFR and NF1 [16-21].

Recently, Lucence Diagnostics released the FibroPhyllo ${ }^{\text {тм }}$ Tissue Test [22], developed from a 5-gene reverse transcription-PCR assay (which measures the expression of $A B C A 8, A P O D, C C L 19, F N 1$ and PRAME) [12], to augment the pathological distinction between FAs and PTs in breast lumps. Working on the common genes involved across the FEL spectrum, our group developed a 16-gene genomic assay to characterize FELs on core biopsy material using Next Generation Sequencing (NGS) [16]. The genes include the frequently observed MED12 and RARA mutations in both fibroadenomas and phyllodes, as well as the mutations in FLNA, SETD2, KMT2D,TERT promoter, NF1, RB1, TP53, PIK3CA, ERBB4 and EGFR which are seen more in the phyllodes tumors. This 16-gene genomic assay has been used anecdotally in selected cases to aid histopathologically challenging cases, such as the grading of phyllodes tumors [23], and where a malignant spindle cell tumors posed a diagnostic dilemma, mimicking metaplastic breast carcinoma [23, 24]. This novel 16-gene targeted panel [16, 23, 24], curated based on their likely involvement in fibroepithelial tumorigenesis, combined with a predictive model, has the potential to serve as an adjunctive diagnostic tool, refining indeterminate histological diagnoses in FELs, particularly on core biopsies.

\section{Methods \\ Clinical specimens and diagnostic criteria}

This study was conducted with the approval of the Centralized Institutional Review Board (CIRB Ref: 2016/2819). A total of 275 FEL specimens, comprising 241 core biopsies and 34 surgical excisions, was randomly selected from cases diagnosed at the Department of Anatomical Pathology, Singapore General Hospital from 2008 to 2012. Twentyfour FELs were from 12 paired specimens, each comprising a core biopsy and a surgical excision removed from the same patient. Haematoxylin and eosin (H\&E)-stained slides of formalin-fixed and paraffin-embedded (FFPE) samples were retrieved, pathologically examined and graded in accordance to recommendations of the World Health Organization Classification of Tumors of the Breast [25]. Examples of the histological features of each subtype are as shown in Fig. 1.

\section{DNA extraction and quality control}

Core biopsies and representative areas of resected tumors were identified and cut into two $10-\mu \mathrm{m}$-thick sections. Deparaffinization of sections was carried out with changes of limonene and graded ethanol. These deparaffinized sections were subsequently air dried and used for genomic DNA (gDNA) extraction with the QIAamp DNA FFPE Tissue Kit (Qiagen, Germany) as per protocol. PicoGreen fluorometric analysis and multiplex PCR assay were used to determine the quantity and quality of the extracted gDNA respectively.

\section{Library preparation for downstream next generation sequencing}

Amplicon-based sequencing libraries were prepared in accordance to the manufacturer's instructions within the QIAseq Targeted DNA Panel Kit (Qiagen, United 


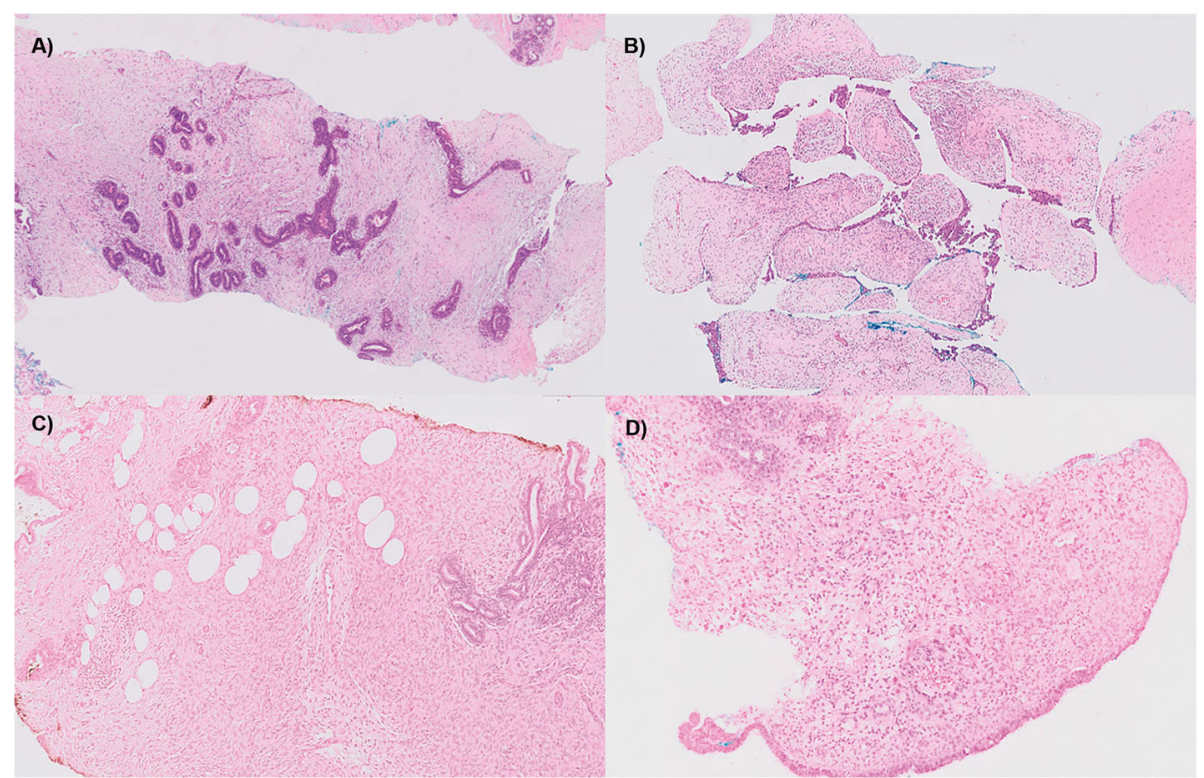

Fig. 1 Histological features of representative core needle biopsies of a) fibroadenoma, b) benign, c) borderline and d) malignant phyllodes tumors confirmed on subsequent excisions

States), using a minimum of $50 \mathrm{ng}$ of gDNA. The gDNA was fragmented prior to the ligation with QIAseq adapters. Post ligation, amplification of the ligated products was carried out using the custom panel comprising of 16 genes-MED12, TERT promoter (Chr 5: 1295100-1,295,300), KMT2D, RARA, SETD2, FLNA, NF1, EGFR, RB1, TP53, PIK3CA, BCOR, PTEN, ERBB4, MAP3K1 and IGF1R [16, 23, 24]. The amplified prepared library was subsequently sequenced to a depth of greater than $300 \mathrm{X}$ of the target regions on the Illumina Hiseq 4000, to generate 150 bp pairedend reads.

\section{Bioinformatics analysis and the validation of variants}

A quality assessment of the raw reads was done using FastQC (version 0.11.5). The trimmed reads were obtained after removing the first $30 \mathrm{bp}$ of reads (adapters) and were aligned to the human genome, hg 19, using BWA-MEM (version 0.7.15-r1140, default setting). Point mutations and indels were called using FreeBayes (version v1.1.0-4-gb6041c6, settings: -m30 -q30 -F0.01) and annotated with wANNOVAR (http://wannovar.wglab.org/). Variants were further filtered to remove synonymous variants and variants in dbSNP. Variants that did not have a minimum coverage of 100X or have allelic frequencies lower than $5 \%$ were excluded. The remaining variants were visually curated using the Integrative Genomics Viewer (IGV) 2.3 genome browser to further exclude possible germline mutations and sequencing artifacts [26]. The data has been uploaded on EMBL-EBI, European
Nucleotide Archive (https://www.ebi.ac.uk/ena); ascension number: PRJEB34134.

\section{Statistical analyses}

Demographics were reported using $\mathrm{T}$ Test and Fisher's Exact Test for numerical and categorical variables respectively. The frequencies of mutations and types of mutations observed in the 16 genes were compared between FAs and the PTs using the Fisher's Exact Test. The number of mutations observed in each sample, defined hereafter as the mutation count, were tabulated and the samples were subsequently categorized into two groups-three or more mutations and less than three mutations. Similarly, these two groups were compared using the Fisher's Exact Test.

\section{The development of a predictive scoring system}

Univariate logistic regression models were generated to determine the variables to be used in the construction of a multivariate logistic regression model. Variables were only included when their $p<0.10$. The multivariate logistic regression model was built using backward selection. Predictors which attained $p<0.05$ were subsequently included the final model. The beta coefficients of these predictors in the final model were transformed into risk scores by dividing the coefficients by the lowest value. The performance of this multivariate model was assessed by the area under the Receiver Operating Characteristics (AUROC) curve and Hosmer Lemeshow Goodness of Fit Test. The risk groupings were further assessed in a logistic regression model using the true diagnoses as an outcome. Statistical significance was 
defined as when $p<0.05$. An internal validation of our prognostic model was performed using the Bootstrapping technique. All analyses were performed in Stata version 12.0 [27].

Five other classification models were attempted to determine the best prediction model and to develop a predictive scoring system - gradient boosting, random forest, decision tree, support vector machine and $\mathrm{KN}$ neighbour classifier. 10-fold cross validations for each model were performed and the accuracy score for each model was calculated using python.

\section{Results}

\section{Demographics of the patients and characteristics of breast Fibroepithelial lesions}

A total of 275 diagnosed FELs-212 FAs, 35 benign, 21 borderline and 7 malignant PTs-were analyzed in this study. Of these, 12 were paired biopsies (i.e. core biopsy and surgical excision from the same patient) -5 FAs, 3 benign, 2 borderline and 2 malignant PTs.

The mean ages of the 207 patients diagnosed with FAs and 56 patients diagnosed with PTs were 45.2 (standard deviation, $\mathrm{SD}=12.3)$ and 47.6 years $(\mathrm{SD}=11.9)$ old respectively. The higher than expected mean age of diagnosis of FAs in this study is likely due to a clinical and selection bias, where older women with breast lumps are preferentially selected to undergo a biopsy or an excision, in contrast to observation and active surveillance in the younger population. There were no significant differences observed between these two groups of patients with respect to age $(p=0.189)$ and ethnicity $(p=0.101$; Table 1$)$.

\section{An assessment of the quality and quantity of the extracted DNA}

DNA was extracted from the FFPE tissues of all 275 FELs, but only $74.3 \%(n=179)$ and $94.1 \%(n=32)$ of the 241 core biopsies and 34 surgical excisions, respectively, contained DNA of sufficient quality and quantity for targeted sequencing (Fig. 2a). As observed, the quality of the extracted gDNA tended to suffer as the number of archival years of the FFPE tissues increased. Excluding the 2008 samples, we achieved a high success rate of
81.3\% in extracting good quality DNA of sufficient quantity from core biopsy specimens. Hence, good quality DNA that is suitable for downstream NGS, was effectively obtained from FFPE samples that were less than 7 to 8 years of age (Fig. 2b). Although there was a higher success rate of extracting good quality amplifiable DNA observed with surgical excision specimens, the majority of them were derived from recent years (Fig. 2c).

Of the 211 FELs that passed the quality and quantity assessment to proceed with downstream NGS were 167 FAs, 24 benign, 14 borderline and 6 malignant PTs. Amongst them were three paired biopsies that included a benign PT and two malignant PTs.

\section{Molecular profiling of the breast Fibroepithelial lesions}

A total of 321 mutations were seen in 164 samples, encompassing 125 FAs, 20 benign, 13 borderline and all 6 malignant PTs. No mutations were seen in the remaining 47 FELs, which included 42 FAs, 4 benign and 1 borderline PT (Fig. 3).

Mutations were observed in all 16 genes across the FELs, except for a lack of PTEN mutations in FAs and an absence of MAP3K1 and IGF1R mutations in PTs. Common to all grades of PTs were mutations in $M E D 12$, TERT promoter, FLNA and RB1. In both benign and borderline PTs, mutations in KMT2D, RARA, SETD2 and PIK3CA were present. Mutations in NF1 and TP53 were observed in both benign and malignant PTs. Common to both borderline and malignant PTs were mutations in EGFR. A mutation in ERBB4 was seen in the benign PTs only; whereas, mutations in $B C O R$ and PTEN were observed in the borderline PTs only (Fig. 3).

\section{Concordance of the molecular profiles of the paired biopsies}

Although there were 12 paired biopsies retrieved for this study, only 3 pairs were suitable for analysis. Of these three pairs, only one pair of malignant PTs had an identical genetic profile in both the core biopsy and surgical excision specimens. They had mutations in MED12,

Table 1 Demographics of patients with fibroepithelial lesions in this study

\begin{tabular}{|c|c|c|c|}
\hline Features & Fibroadenoma $(n=207)^{\dagger}$ & Phyllodes Tumor $(n=56)^{+}$ & $p$ value \\
\hline$\overline{\text { Age }(\text { mean, SD) }}{ }^{a}$ & $45.2,12.3$ & $47.6,11.9$ & 0.189 \\
\hline \multicolumn{4}{|l|}{ Ethnicity $(n, \%)^{b}$} \\
\hline Chinese & $152(73.4 \%)$ & $33(58.9 \%)$ & 0.101 \\
\hline Malay & $17(8.2 \%)$ & 10 (17.9\%) & \\
\hline Indian & $10(4.8 \%)$ & $4(7.1 \%)$ & \\
\hline Others & $028(13.5 \%)$ & 09 (16.1\%) & \\
\hline
\end{tabular}

${ }^{t}$ The surgical excisions of the paired biopsies were excluded

${ }^{a}$ T Test, comparing the age distribution between the patients diagnosed with fibroadenomas (FA) and phyllodes tumor (PT)

${ }^{\mathrm{b}}$ Fisher's Exact Test, comparing the ethnicity distribution between the patients diagnosed with FA and PT. 


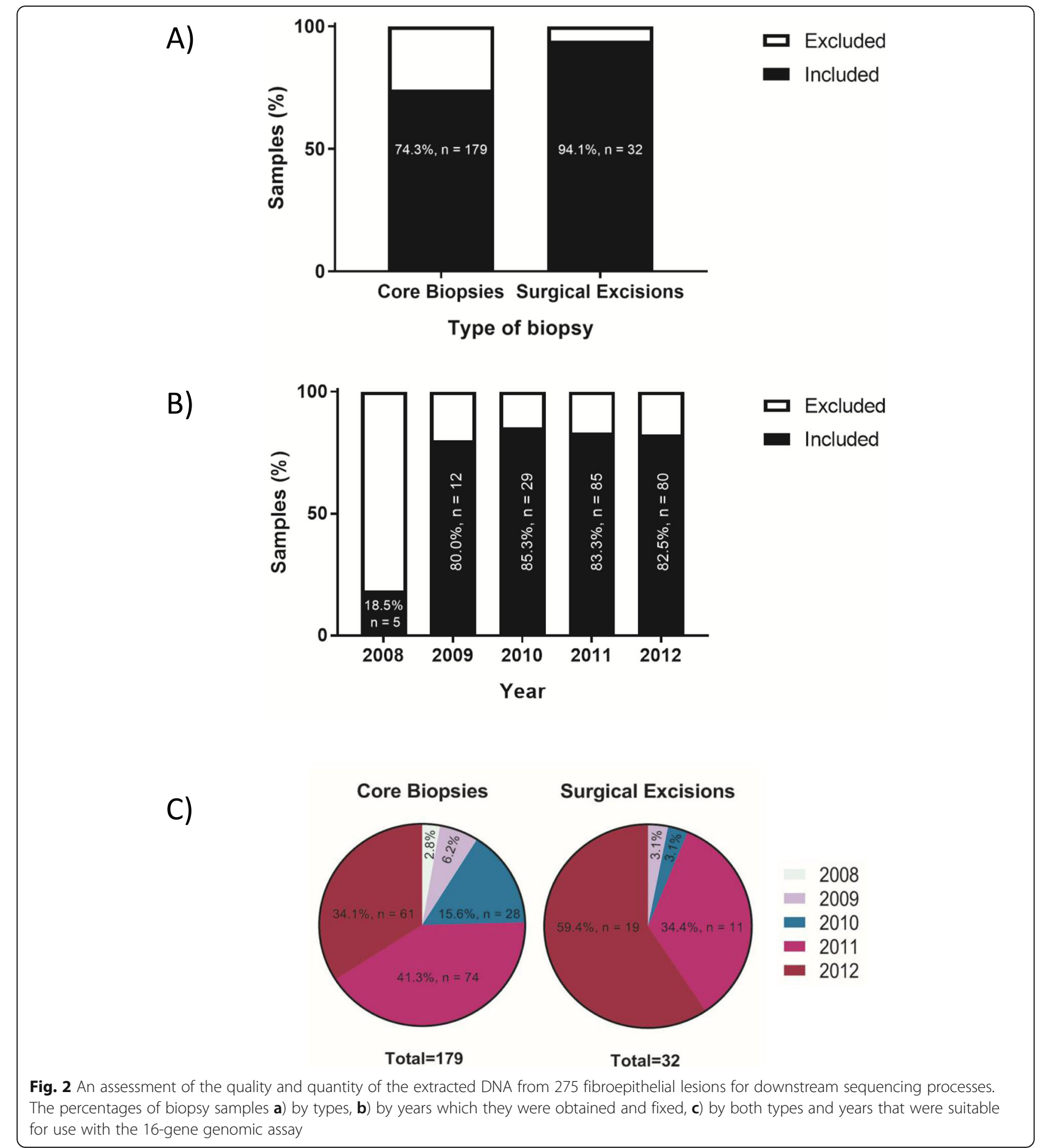

TERT promoter and FLNA (Fig. 3). An analysis of the remaining two other pairs of biopsies showed the following: 1) the first was a pair of benign PTs, with the surgical excision possessing a mutation in KMT2D but with no mutations observed in the core biopsy; 2) the second pair of malignant PTs had mutations in TP53 and RB1 in both samples, but there was an additional mutation in the NF1 gene observed in the core biopsy but not in the surgical excision (Fig. 3).

TERT promoter, RARA, FLNA, RB1 and TP53 were more likely to be mutated in Phyllodes tumors

Most abnormalities occurred in codon 44 of exon 2 in $M E D 12$, the most frequently mutated gene amongst the 


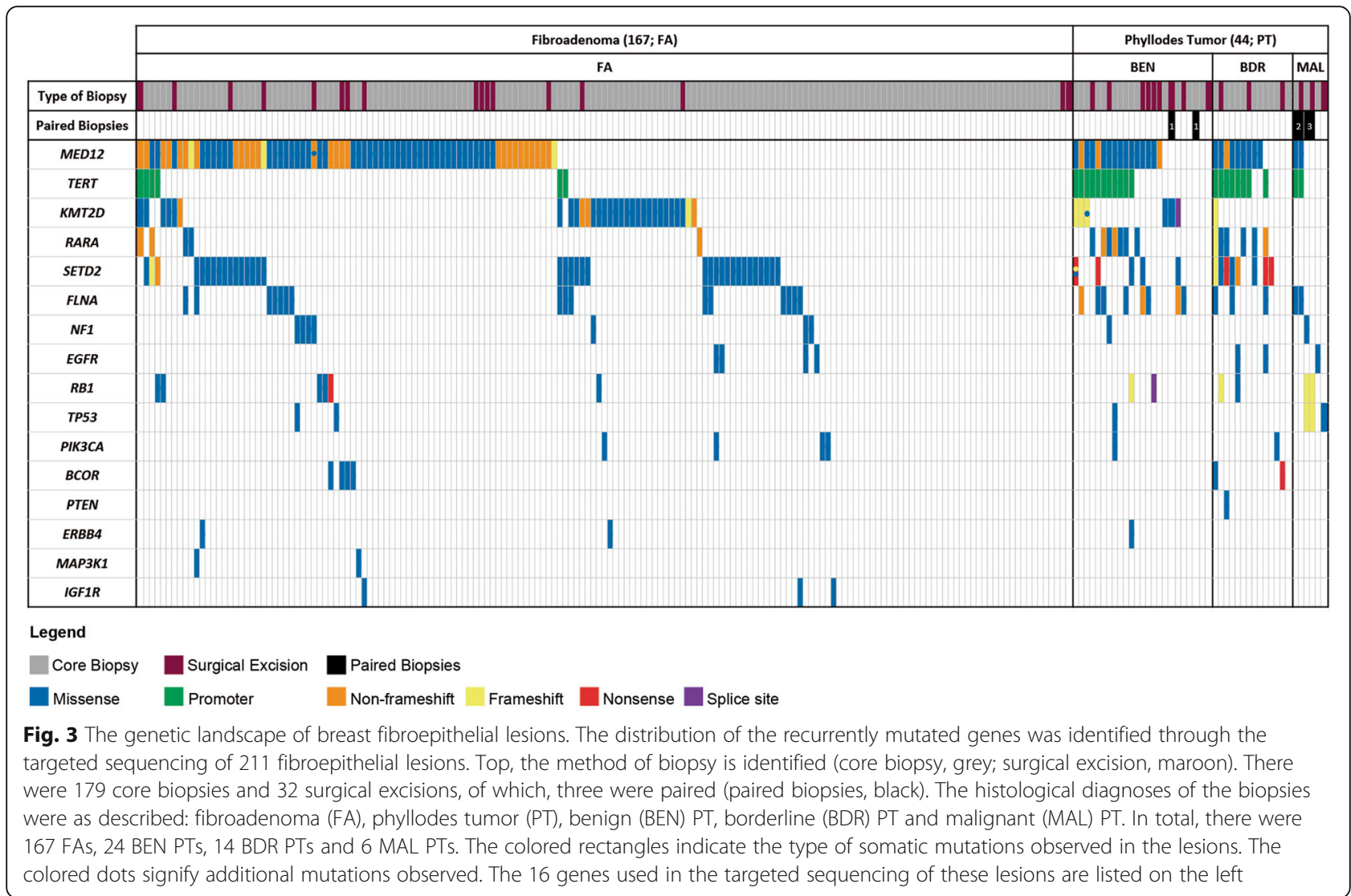

Table 2 Frequencies of mutations in the 16 genes, by FEL subtypes. Benign (BEN), borderline (BDR) and malignant (MAL) phyllodes tumor (PT)

\begin{tabular}{|c|c|c|c|c|c|c|c|c|c|c|c|}
\hline \multirow[t]{3}{*}{ Gene } & \multirow{2}{*}{\multicolumn{2}{|c|}{$\begin{array}{l}\text { Fibroadenoma } \\
(n=167)\end{array}$}} & \multicolumn{8}{|c|}{ Phyllodes Tumor $(n=44)$} & \multirow[t]{3}{*}{$p$ value } \\
\hline & & & \multicolumn{2}{|c|}{$\mathrm{BEN}(n=24)$} & \multicolumn{2}{|c|}{$\operatorname{BDR}(n=14)$} & \multicolumn{2}{|c|}{$\operatorname{MAL}(n=6)$} & \multicolumn{2}{|c|}{ All PT } & \\
\hline & $n$ & (\%) & $n$ & (\%) & $n$ & (\%) & $n$ & (\%) & $n$ & (\%) & \\
\hline MED12 & 75 & $(44.9)$ & 16 & (66.7) & 9 & (64.3) & 2 & (33.3) & 27 & (61.4) & 0.063 \\
\hline TERT & 6 & (3.6) & 11 & (45.8) & 8 & (57.1) & 2 & (33.3) & 21 & $(47.7)$ & $<0.001$ \\
\hline KMT2D & 30 & $(18.0)$ & 6 & (25.0) & 1 & (7.1) & 0 & $(0.0)$ & 7 & (15.9) & 0.827 \\
\hline RARA & 5 & (3.0) & 7 & $(29.2)$ & 6 & $(42.9)$ & 0 & $(0.0)$ & 13 & (29.6) & $<0.001$ \\
\hline SETD2 & 36 & (21.6) & 5 & (20.8) & 8 & (57.1) & 0 & $(0.0)$ & 13 & (29.6) & 0.316 \\
\hline FLNA & 16 & (9.6) & 8 & (33.3) & 3 & (21.4) & 2 & (33.3) & 13 & (29.6) & 0.002 \\
\hline$N F 1$ & 7 & $(4.2)$ & 1 & $(4.2)$ & 0 & $(0.0)$ & 1 & $(16.7)$ & 2 & (4.6) & 1.000 \\
\hline EGFR & 4 & (2.4) & 0 & $(0.0)$ & 2 & (14.3) & 1 & $(16.7)$ & 3 & (6.8) & 0.160 \\
\hline$R B 1$ & 6 & (3.6) & 2 & (8.3) & 2 & (14.3) & 2 & (33.3) & 6 & (13.6) & 0.020 \\
\hline TP53 & 2 & $(1.2)$ & 1 & $(4.2)$ & 0 & $(0.0)$ & 3 & $(50.0)$ & 4 & $(9.1)$ & 0.018 \\
\hline PIK3CA & 4 & (2.4) & 1 & $(4.2)$ & 1 & (7.1) & 0 & $(0.0)$ & 2 & (4.6) & 0.607 \\
\hline$B C O R$ & 4 & (2.4) & 0 & $(0.0)$ & 2 & (14.3) & 0 & $(0.0)$ & 2 & (4.6) & 0.607 \\
\hline PTEN & 0 & $(0.0)$ & 0 & $(0.0)$ & 1 & (7.1) & 0 & $(0.0)$ & 1 & (2.3) & 0.209 \\
\hline ERBB4 & 2 & $(1.2)$ & 1 & $(4.2)$ & 0 & $(0.0)$ & 0 & $(0.0)$ & 1 & (2.3) & 0.506 \\
\hline MAP3К1 & 2 & $(1.2)$ & 0 & $(0.0)$ & 0 & $(0.0)$ & 0 & $(0.0)$ & 0 & $(0.0)$ & 1.000 \\
\hline IGFIR & 3 & (1.8) & 0 & $(0.0)$ & 0 & $(0.0)$ & 0 & $(0.0)$ & 0 & $(0.0)$ & 1.000 \\
\hline
\end{tabular}


FELs. MED12 mutations were observed in 44.9 and $61.4 \%$ of FAs and PTs, respectively. The percentages of samples that exhibited MED12 mutations were observed to decrease across the PT grades-66.7, 64.3 and 33.3\% in benign, borderline and malignant PTs respectively (Table 2).

The TERT promoter was the next most commonly mutated gene in PTs (Table 2). TERT promoter mutations were rare in FAs. In PTs, a mutation in the TERT promoter was often accompanied by a mutation in the MED12 gene. Despite that, two FAs and a single PT displayed a mutation in the TERT promoter without a mutation in the MED12 gene. Another set of 16 PTs -8 benign, 4 borderline and 4 malignant PTs lacked mutations in both MED12 and the TERT promoter. However, these PTs had additional mutations in KMT2D, SETD2, FLNA, PIK3CA, BCOR, NF1, EGFR, RB1 and TP53 (Fig. 3).

There were significantly higher mutation rates in PTs than FAs in these genes-the TERT promoter $(47.7 \%$ in PTs vs $3.6 \%$ in FAs; $p<0.001), R A R A$ (29.6\% in PTs vs $3.0 \%$ in FAs; $p<0.001$ ), FLNA (29.6\% in PTs vs $9.6 \%$ in FAs; $p=0.002$ ), RB1 (13.6\% in PTs vs $3.6 \%$ in FAs; $p=0.020)$ and TP53 (9.1\% in PTs vs $1.2 \%$ in FAs; $p=0.018$; Table 2 ).

\section{Phyllodes tumors have significantly higher mutation counts than Fibroadenomas}

Of the total of 321 genetic alterations observed in our study, a total of 203 mutations were from FAs, and $118 \mathrm{mu}-$ tations were from PTs (Fig. 3). PTs possessed significantly higher mutation counts than FAs, with a higher percentage of PTs having three or more mutations (54.6\% in PTs vs $8.4 \%$ in FAs; $p<0.001$ ) compared to FAs (Table 3). The FAs had a median of 1 mutation (mean $=1.2$, range $=0$ to 4 ), and the PTs had a median of 3 mutations (mean $=2.7$, range $=0$ to 7 ). However, there were no significant differences in the number of mutations observed between benign, borderline and malignant PTs $(p=1.000)$, implying that either the number of mutations gained may not have any implications on PT grade, or the sample size is too small which is likely the case.

\section{Types of mutations observed in breast Fibroepithelial lesions}

Applying our 16-gene assay on our samples, the following mutations were observed in FAs: missense, promoter, non-

Table 3 Fibroepithelial lesions were classified into two groups: 1) three or more mutations or 2) less than three mutations. The two groups were compared using the Fisher's Exact Test

\begin{tabular}{|c|c|c|c|c|c|}
\hline \multirow{2}{*}{$\begin{array}{l}\text { Mutation } \\
\text { count }\end{array}$} & \multicolumn{2}{|c|}{ Fibroadenoma $(n=167)$} & \multicolumn{2}{|c|}{ Phyllodes Tumor $(n=44)$} & \multirow[t]{2}{*}{$p$ value } \\
\hline & $n$ & $\%$ & $n$ & $\%$ & \\
\hline$<3$ & 153 & 91.6 & 20 & 45.5 & $<0.001$ \\
\hline$\geq 3$ & 14 & 8.4 & 24 & 54.6 & \\
\hline
\end{tabular}

frameshift, frameshift and nonsense mutations. The following mutations were observed in PTs: missense, promoter, non-frameshift, frameshift, nonsense and splice site mutations (Fig. 3).

From the 167 FAs and 44 PTs sequenced, we discovered that missense mutations were the most common in both FAs and PTs (65.9\% in PTs vs $77.3 \%$ in FAs). There were significantly more PTs having promoter mutations (47.7\% in PTs vs 3.6\% in FAs; $p<0.001$ ), frameshift mutations $(18.2 \%$ in PTs vs $3.0 \%$ in FAs; $p=0.001$ ), nonsense mutations $(13.6 \%$ in PTs vs $0.6 \%$ in FAs; $p<0.001)$ and splice site mutations $(4.6 \%$ in PTs vs $0 \%$ in FAs; $p=0.043$; Table 4).

\section{A predictive scoring system as an adjunctive diagnostic tool for FELs}

The univariate analyses identified ten predictors with $p<$ 0.10: mutations in MED12, TERT promoter, $R A R A$, FLNA, RB1, TP53; promoter, frameshift and nonsense mutations, as well as the possession of three or more mutations (Table 5). These ten variables were subsequently used to build a multivariate logistic regression model. The variables with $p<0.05$ in the final model were mutations in TP53 (OR $=13.54,95 \%$ confidence interval, CI: 2.99 to 61.31$)$, TERT promoter $(\mathrm{OR}=24.10$, 95\% CI: 10.94 to 53.10$)$ and nonsense mutation (OR = 19.75, 95\% CI: 5.32 to 73.30; Table 6). The ROC of the model was $0.773,95 \% \mathrm{CI}: 0.70$ to 0.85 , which showed that this model had good discriminant ability. The Hosmer-Lemeshow Goodness of Fit Test indicated good calibration with $p=0.945$. An internal validation of our prognostic model using the bootstrapping technique was carried out based on 796 replications providing the same coefficient estimates with $\mathrm{ROC}=0.81,95 \% \mathrm{CI}(0.72$ to 0.90). This demonstrates good discriminant ability between FA and PT, and the Hosmer Lemeshow goodness of fit test $p$-value was 0.973 , indicating good calibration.

In addition to the logistic regression model, five other classification models were applied to determine the best prediction model for a predictive scoring system. These include gradient boosting, radnom forest, decision tree, support vector machine and $\mathrm{KN}$ neighbour classifier. A 10 fold cross validation was performed for all 6 models, and the accuracy scores were calculated and compared (Table 7). With the highest accuracy score, the logistic regression model was then adopted in our prediction model.

The beta coefficients of these chosen predictors in the final model were used to calculate the risk scores. Presence of TP53 mutation, promoter and nonsense mutations each contributed 1, 1.22 and 1.14 points, respectively. The risk score was classified into two groups: i) low risk ( $<1$ point) and ii) high risk $(\geq 1$ point) of being a PT (Table 8). Logistic regression 
Table 4 Types of mutations observed in 211 fibroepithelial lesions

\begin{tabular}{|c|c|c|c|c|c|}
\hline \multirow{2}{*}{$\begin{array}{l}\text { Type of } \\
\text { Mutation }\end{array}$} & \multicolumn{2}{|c|}{ Fibroadenoma $(n=167)$} & \multicolumn{2}{|c|}{ Phyllodes Tumor $(n=44)$} & \multirow[t]{2}{*}{$p$ value } \\
\hline & $\bar{n}$ & (\%) & $\bar{n}$ & (\%) & \\
\hline Missense & 110 & (65.9) & 34 & (77.3) & 0.202 \\
\hline Promoter & 6 & (3.6) & 21 & $(47.7)$ & $<0.001$ \\
\hline Non Frameshift & 33 & (19.8) & 10 & $(22.7)$ & 0.676 \\
\hline Frameshift & 5 & (3.0) & 8 & $(18.2)$ & 0.001 \\
\hline Nonsense & 1 & $(0.6)$ & 6 & (13.6) & $<0.001$ \\
\hline Splice site & 0 & $(0.0)$ & 2 & (4.6) & 0.043 \\
\hline
\end{tabular}

analysis showed that these groupings significantly predicted PTs with $p<0.001$; those in the high risk group were 25 times more likely to have PT compared to the low risk group.

The diagnoses of all 211 lesions obtained through the predictive model were subsequently compared against the pathological reports, and 27 discordant cases were observed-9 FAs and 18 PTs on the original pathological reports, but were predicted otherwise by the 16-gene panel assay and the predictive scoring system (Fig. 4). Six of these nine FAs that were identified as PTs by the model, had possessed a TERT promoter mutation and other mutations in

Table 5 A summary of the univariate analyses performed to understand the effect of each potential predictor in the classification of the fibroepithelial lesions. Predictors $\left({ }^{\dagger}\right)$, with $p<0.10$, were included in the multivariate analysis

\begin{tabular}{|c|c|c|c|}
\hline Predictors & Odds Ratio (OR) & 95\% Confidence Interval (Cl) & $p$ value \\
\hline \multicolumn{4}{|l|}{ Genes } \\
\hline MED12 ${ }^{+}$ & 1.95 & 0.99 to 3.84 & 0.054 \\
\hline TERT promoter ${ }^{t}$ & 24.50 & 8.95 to 67.07 & $<0.001$ \\
\hline KMT2D & 1.10 & 0.46 to 2.60 & 0.836 \\
\hline$R A R A^{+}$ & 13.59 & 4.52 to 40.84 & $<0.001$ \\
\hline SETD2 & 1.53 & 0.72 to 3.22 & 0.266 \\
\hline$F L N A^{+}$ & 3.96 & 1.73 to 9.06 & 0.001 \\
\hline NF1 & 1.09 & 0.22 to 5.43 & 0.918 \\
\hline EGFR & 2.98 & 0.64 to 13.85 & 0.163 \\
\hline$R B 1^{+}$ & 4.24 & 1.29 to 13.86 & 0.017 \\
\hline $\operatorname{TP}^{+} 3^{+}$ & 8.25 & 1.46 to 46.64 & 0.017 \\
\hline PIK3CA & 1.94 & 0.34 to 10.96 & 0.453 \\
\hline$B C O R$ & 1.94 & 0.34 to 10.96 & 0.453 \\
\hline PTEN & \multicolumn{3}{|l|}{ Excluded, frequency $<5$} \\
\hline ERBB4 & 1.92 & 0.17 to 21.66 & 0.598 \\
\hline MAP3К1 & \multicolumn{3}{|l|}{ Excluded, frequency $<5$} \\
\hline IGFIR & \multicolumn{3}{|l|}{ Excluded, frequency $<5$} \\
\hline \multicolumn{4}{|l|}{ Mutation types } \\
\hline Missense & 1.76 & 0.81 to 3.82 & 0.152 \\
\hline Promoter $^{\dagger}$ & 24.50 & 8.95 to 67.07 & $<0.001$ \\
\hline Non Frameshift & 1.19 & 0.54 to 2.67 & 0.664 \\
\hline Frameshift $^{\dagger}$ & 7.20 & 2.23 to 23.30 & 0.001 \\
\hline Nonsense $^{t}$ & 26.20 & 3.06 to 224.10 & 0.003 \\
\hline Splice site & \multicolumn{3}{|l|}{ Excluded, frequency $<5$} \\
\hline \multicolumn{4}{|l|}{ Mutation count } \\
\hline Mutation count $(\geq 3)^{+}$ & 13.11 & 5.85 to 29.40 & $<0.001$ \\
\hline
\end{tabular}


Table 6 A summary of the predictors whose $p<0.05$ that were included in the final multivariate logistic regression model

\begin{tabular}{|c|c|c|c|c|c|}
\hline Predictors & Odds Ratio (OR) & $\begin{array}{l}\text { 95\% Confidence } \\
\text { Interval (Cl) }\end{array}$ & $\beta$ coefficient & Standard Error (SE) & $p$ value \\
\hline \multicolumn{6}{|l|}{ Genes } \\
\hline TP53 & 13.54 & 2.99 to 61.31 & 2.61 & 0.94 & 0.001 \\
\hline \multicolumn{6}{|c|}{ Mutation types } \\
\hline Promoter & 24.10 & 10.94 to 53.10 & 3.18 & 0.63 & $<0.001$ \\
\hline Nonsense & 19.75 & 5.32 to 73.30 & 2.98 & 0.77 & $<0.001$ \\
\hline
\end{tabular}

other genes such as FLNA, KMT2D, MED12, RARA, RB1 and SETD2 (Fig. 4). Subsequent pathological review of these nine FAs yielded an upgrade of two FAs (Sample \#52 and \#114) to benign PTs (Figs. 5a, b, and c). While three samples (\#8, \#73 and \#178) were also noted to have increased cellularity, their original pathological diagnoses of FAs were upheld. In addition, a clinical follow up of these nine patients showed clinical and radiological stability for at least two years, thereby providing support to the original histological diagnoses of FAs. The only exception was the patient (with sample \#114) who had a recurrence of a benign PT, and this is concordant with the revised pathological diagnosis. On the contrary, the 18 lesions that were diagnosed as PTs in the pathological reports, were reported as FAs by our predictive model due to the lack of TP53 mutations and/or a promoter and/or a nonsense mutation. However, they had mutations in other genes such as EGFR, FLNA, KMT2D, MED12, RARA, RB1, SETD2 and PIK3CA (Fig. 4). Subsequent pathological review of these PTs yielded a downgrade of three PTs (Sample \#63, \#105 and $\# 168$ ) to FAs (Figs. 5d, e, f and g). Together, the 16-gene assay and the predictive model had an accuracy of $89.6 \%$, a specificity of $95.8 \%$, a sensitivity of $65.1 \%$, a positive predictive value (PPV) of $80.0 \%$ and a negative predictive value (NPV) of $91.5 \%$.

\section{Discussion}

In this study, we successfully extracted DNA of good quality and quantity from archival FFPE specimens of up to ten years of age, despite the known damage of long term formalin fixation to DNA. Formaldehyde, the main constituent of formalin, is known to generate crosslinks between intracellular macromolecules, forming protein-

Table 7 A comparison of the accuracy scores across the 6 different classification models (statistical and machine learning techniques)

\begin{tabular}{ll}
\hline Model & Accuracy Score \\
\hline Logistic Regression & 0.87 \\
Gradient Boosting & 0.85 \\
Random Forest & 0.82 \\
Decision Tree & 0.76 \\
Support Vector Machine & 0.77 \\
\hline
\end{tabular}

protein, protein-DNA structures, DNA-formaldehyde adducts and inter-strand DNA crosslinks. DNA fragmentation has also been observed to worsen with the lower $\mathrm{pH}$ of formalin used during tissue fixation and the longer storage period of FFPE specimens. Formaldehyde also readily oxidizes with the atmospheric oxygen to form formic acid, encouraging the formation of abasic sites in DNA. Formalin fixation causes cytosine deamination which results in the formation of $\mathrm{C}>\mathrm{T}$ and $\mathrm{G}>\mathrm{A}$ sequencing artefacts, thereby leading to the increase in single-nucleotide variant (SNV) rate [28-31]. Hence, to minimize sequencing artefacts that could complicate analysis, strict criteria in the preparation and quality control of FFPE extracted DNA had to be observed, using only biopsies with good quality DNA of sufficient quantity for downstream NGS. Good quality DNA was extracted from $81.3 \%$ of the core biopsies and $94.1 \%$ of the surgical excisions (Fig. 2a). Although it appears that surgical excisions were a better source of good quality DNA, most of the surgical excisions were "newer" FFPE tissues and hence could have been subjected to lesser

Table 8 The scorecard describing the weightage points of each predictor that was derived through their beta coefficients and the cut-off points required for a lesion to be classified as either a fibroadenoma or a phyllodes tumor

\begin{tabular}{lc}
\hline Predictors & Score \\
\hline Genes & \\
Presence of mutations in TP53 gene & 1 \\
Yes & 0 \\
No & \\
Mutation types & \\
Presence of promoter mutation & 1.22 \\
Yes & 0 \\
No & \\
Presence of nonsense mutation & 1.14 \\
Yes & 0 \\
No & \\
Risk groups & $<1$ \\
Low risk of being a phyllodes tumor & \\
High risk of being a phyllodes tumor
\end{tabular}




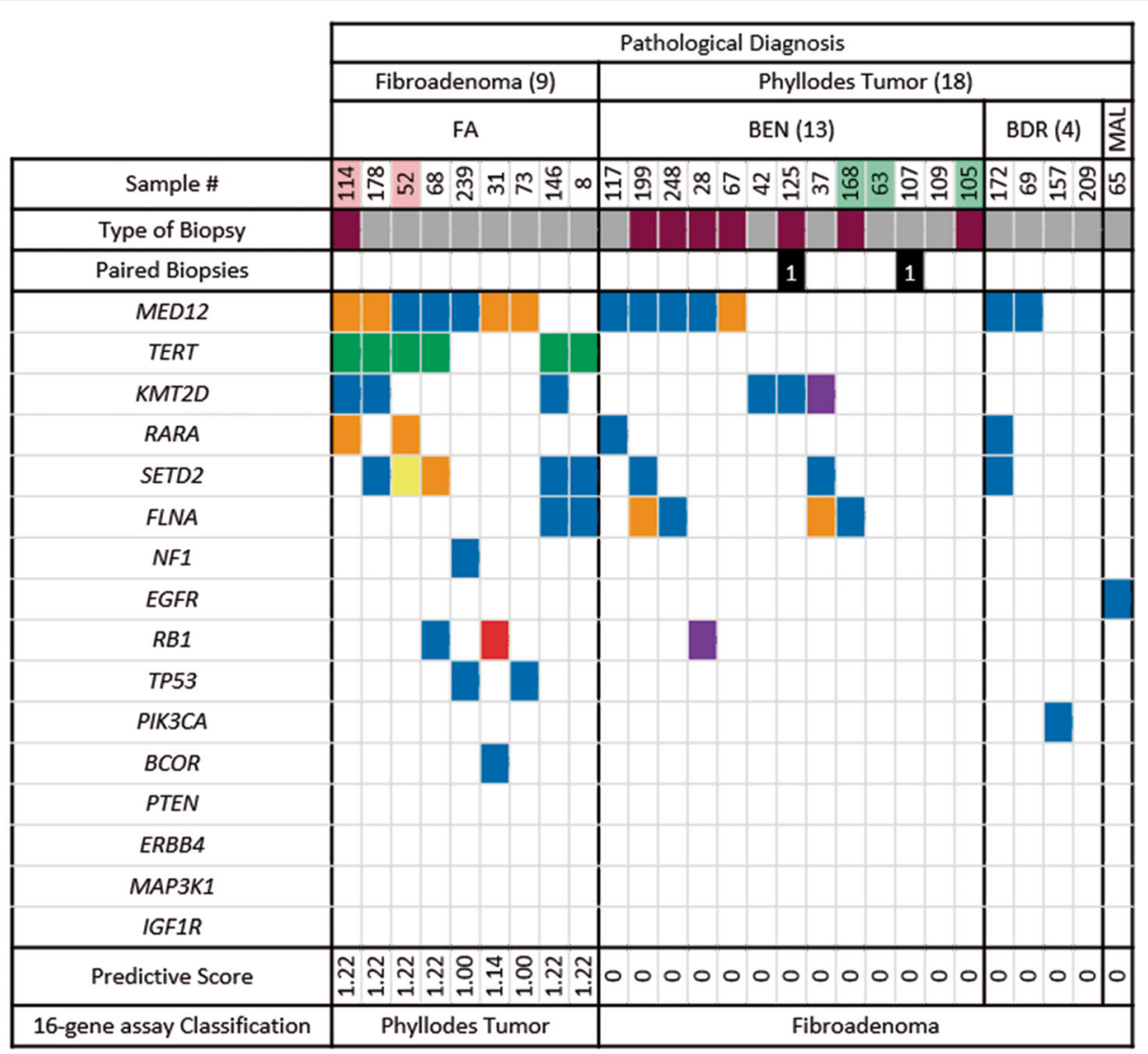

\section{Legend}

\section{Core Biopsy}

Missense
Surgical Excision

Promoter
Paired Biopsies Fibroadenoma (after review)

Non-Frameshift Frameshift
Nonsense
Phyllodes Tumor (after review)

Splice site

Fig. 4 The genetic landscape of the 27 discordant cases, comprising 9 fibroadenomas (FAs) and 18 phyllodes tumors (PTs). This figure demonstrates the discrepancies between the original pathological diagnoses of the FELs and the diagnoses of the fibroepithelial lesions based on the predictive scoring model and the 16-gene assay. Top, pathological diagnoses, the sample identification number and type of biopsy material. Samples that have their diagnoses changed on second pathology review are highlighted. Left, the 16 genes used in the targeted sequencing of these lesions are listed. The colored rectangles indicate the type of somatic mutations observed in the lesions through the 16gene assay. Bottom, the respective predictive scores calculated based on the scorecard (Table 6) and their corresponding classifications by the 16-gene assay

degradation by formalin (Fig. 2c). Furthermore, the surgical excisions were larger than core biopsies, offering greater amounts of tissue for gDNA extraction [30]. With minimal DNA degradation expected from 'fresher' specimens, a greater yield of DNA with good quality and quantity from an even smaller volume of tissue should be expected for successful downstream application of NGS.

In this study, MED12 was identified as the most frequently mutated gene amongst FELs (Table 2) [15, 16, 32-36], with observed frequencies similar to those reported in our previous studies $[13,14]$. The presence of a common mutation in the MED12 gene in both FAs and PTs suggests that they share a similar origin, with the comparable frequencies emphasizing their close molecular relationship [14, 32], and that MED12 aberrations occur early in the pathogenesis of these tumors.

Mutations in the TERT promoter were the second commonest mutation observed in PTs but were rare in FAs (Table 2) [17]. Our study showed a seemingly increasing trend from benign to borderline PTs, suggesting that TERT promoter mutations may drive the progression of PTs [18]. However, this increasing trend did not continue beyond the borderline PTs to the malignant PTs. This may be due to a bias of the small number of malignant PTs studied, and the possibility that a subset of malignant PTs derived from "TERT mutation negative precursors" were included in this analysis [17]. Borderline and malignant PTs of these "TERT mutation negative precursors" lacked mutations in the MED12 gene 


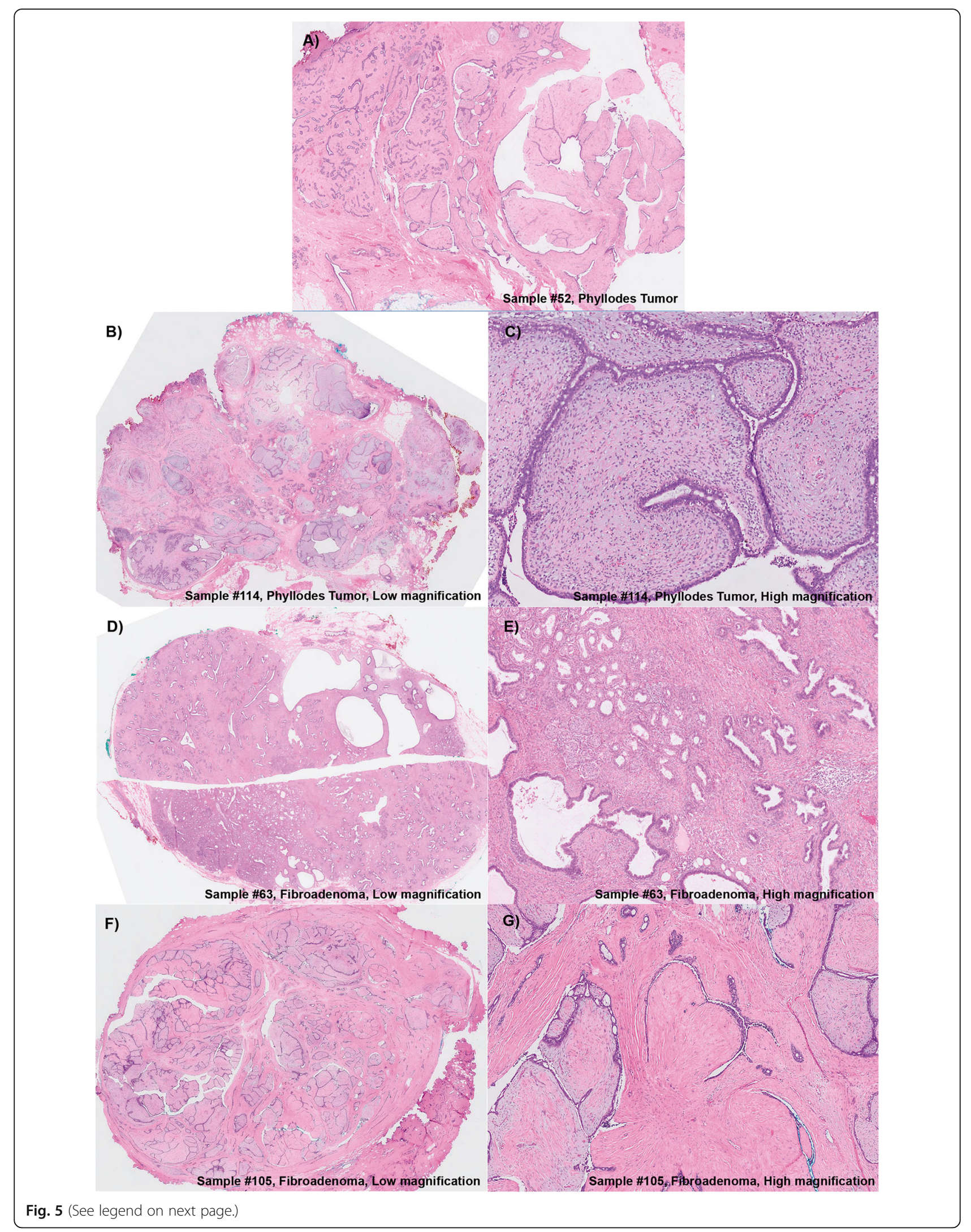




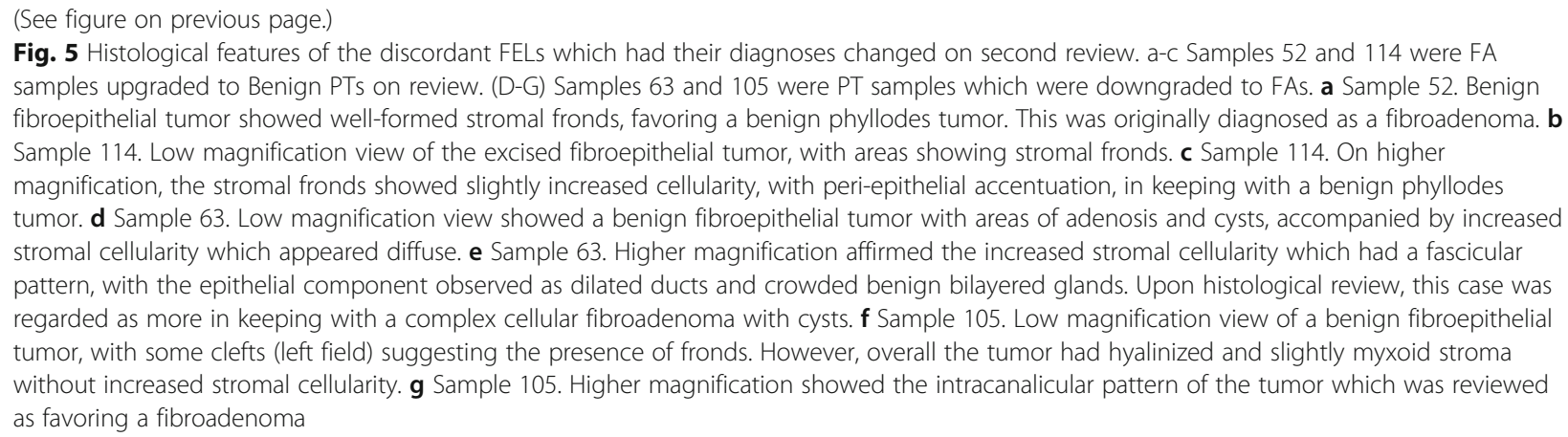

but mutations were present in SETD2, PIK3CA, BCOR, NF1, EGFR, RB1 and TP53 instead (Fig. 3). It is possible that these PTs arose de novo, via a MED12 independent pathway, and that these additional mutations were sufficient for acquisition of higher grade PT phenotypes, even in the absence of mutations in both MED12 and TERT promoter [15, 17, 37].

In addition to the TERT promoter, mutations in RARA, FLNA, RB1 and TP53 were more frequently observed in PTs than FAs (Table 2), supporting the notion that these genes could drive further progression of FAs to PTs, and may be useful in the discrimination of the two entities [17]. Furthermore, from this select 16-gene assay, a significantly greater number of mutations was observed in PTs compared to FAs (Table 3), consistent with the theory of a stepwise progression of FELs [37]. We were unable to demonstrate a significant difference in the number of mutations observed amongst the different PT grades due to the small number of PTs studied. However, from a clinical perspective, the utility in differentiating FAs from PTs (regardless of the grade of PTs) from a core biopsy is a very useful first step in facilitating the surgeon's approach to managing a patient with a breast FEL.

The types of mutations found in PTs differed from the FAs. Apart from the missense mutations that were commonly found across all FELs, PTs were more frequently associated with 'damaging' mutation types, such as the promoter, frameshift, nonsense and splice site mutations (Table 4). We surmise that these mutation types may have more deleterious effects on protein functions and downstream pathways. Though the relationship between the types of mutations and the malignant potential of a tumor is unclear [33], it is possible that both the genes involved and the types of genetic mutations observed may work synergistically in FEL pathogenesis.

Despite the small numbers of paired biopsies analyzed, we have gained a few insights. Mutations in the paired biopsies were fairly concordant (Fig. 3), validating the 16-gene panel on core biopsy specimens with their corresponding surgical excisions. Though the amount of
gDNA available from the core biopsies was limited, we were able to identify additional mutations in two of three paired biopsies. In one pair, the additional mutation (NF1) observed in the core biopsy of a malignant PT was present in its corresponding surgical excision, but the variant allelic frequency of this mutation fell beneath the specified threshold of 5\% and was excluded. This suggests that the assay was sufficiently sensitive in identifying mutations present in core biopsies that might otherwise be missed in a larger tumor specimen. On the contrary, the other paired biopsies of a benign PT had an additional mutation (KMT2D) in the surgical excision specimen and not in the core biopsy (Fig. 3). This additional mutation present in the surgical excision could be due to the core biopsies reflecting only a small part of the tumor and may under-represent the clonal composition of a heterogeneous tumor [38]. The possibility of under-sampling from a core biopsy is a known caveat, and it emphasizes the importance for the clinician in submitting core biopsies that are a good representation of the entire tumor. Due to the retrospective nature of this study and the clinical management of FELs (wide excisions for PTs and surveillance/enucleation for FAs), the procurement of surgical excisions and matched normal tissues is limited for FAs, precluding a more robust genomic comparison between core and excision biopsy material.

Statistical analyses have narrowed down a list of potential predictors that are useful in constructing a predictive scoring system capable of differentiating PTs from FAs. A multivariate regression model was performed on these top ten predictors, identifying three that could work together to classify the FELs. However, with only three predictors in the final model, the scores of these FELs were rather homogenous. It is likely that this relatively small sample size of PTs, a reflection of its low incidence in the clinical setting, may have resulted in the exclusion of other predictors in the final model, due to the lack of significance in multivariate analysis. Nevertheless, the logistic regression results had shown that this predictive model is still competent in predicting PTs $(p<0.001)$. 
Our 16-gene assay and predictive model are comparable to the recently available, commercially developed, molecular FibroPhyllo ${ }^{\text {ma }}$ Tissue Test $[12,39]$, with an accuracy of $89.6 \%$ (vs $92.6 \%$ with FibroPhyllo ${ }^{\mathrm{Tm}}$ Tissue Test), a specificity of $95.8 \%$ (vs $94.7 \%$ ), PPV of $80.0 \%$ (vs 77.3\%) and NPV of $91.5 \%$ (vs 96.2\%) [12]. Despite the lower sensitivity of our model $65.1 \%$ (vs $82.9 \%$ ), this 16 gene panel has acknowledged the biological relevance to FEL pathogenesis, identified and assisted in the reclassification of five FELs. This demonstrates its potential as an adjunctive diagnostic tool in clinical practice, not just in post-operative specimens $[16,23,24]$, but particularly pre-operatively, in those core biopsies with indeterminate diagnoses. In addition, this 16 gene panel assay gives detailed information on the genetic mutations present within the tumour, and hence has potential to help differentiate between the different grades of Phyllodes tumours. Validation of this assay on a larger population of PTs is required, and the possible inclusion of other predictors in the final model may help increase the sensitivity and accuracy.

\section{Conclusion}

To our best knowledge, this is the first study to have successfully demonstrated the application of a novel 16gene assay to reveal mutations across the FEL spectrum on archival FFPE core biopsy specimens. While MED12 remains the most commonly mutated gene amongst FELs, the TERT promoter is the second most frequently mutated gene in PTs. Higher frequencies of mutations observed in the TERT promoter, RARA, FLNA, RB1 and TP53 in PTs emphasized the potential of the 16-gene panel in discriminating FEL subtypes. We have also created a preliminary predictive scoring system that classified FELs on core biopsy with good discrimination and calibration. The predictive scoring system categorizes a FEL at low risk (score $<1$ ) or high risk (score $\geq 1$ ) of being a PT, and is significant in predicting PTs $(p<0.001)$. Further work is needed on a larger number of FELs, particularly PTs, to validate this predictive model. We propose that this gene assay and predictive model have the potential to be used in the clinical setting as an adjunctive tool in the diagnosis of FELs on core biopsies, particularly for indeterminate FELs.

\section{Abbreviations}

BDR: Borderline; BEN: Benign; FA: Fibroadenoma; FEL: Fibroepithelial lesion; FFPE: Formalin-Fixed and Paraffin-Embedded (FFPE); gDNA: Genomic DNA; MAL: Malignant; NGS: Next Generation Sequencing; PT: Phyllodes Tumor

\section{Acknowledgements}

Not Applicable.

\section{Authors' contributions}

YS was the principal investigator, who with the guidance of BTT and PHT, oversaw the project, interpreted the data and predictive model and wrote the manuscript. GXPN, CCYN, VR, SFW,WL extracted the gDNA and ran the genomic assays, GXPN, CCYN, VR, SFW,WL, PG and SN performed the bioinformatics analysis, WYN did the statistical analysis for data and predictive model, AAT, JCTL, NDMN, PHT retrieved, and processed the paraffin samples, and provided histopathology review. VKMT, PM, WSY, CYW, BKTT, KWO obtained and provided tissue specimens. All authors read and approved the final manuscript.

\section{Funding \\ This work was supported by the SingHealth Surgery ACP Surgeon-Scientist Strategic Start-up (S4G) Grant.}

\section{Availability of data and materials \\ The datasets generated and/or analysed during this current study are available from the corresponding author on reasonable request.}

\section{Ethics approval and consent to participate}

This study was conducted with the approval of the SingHealth Centralized Institutional Review Board (CIRB Ref: 2016/2819). A waiver of consent was granted given the retrospective nature of the project, and that archival tissue was used.

\section{Consent for publication}

NA

\section{Competing interests}

None

\section{Author details}

${ }^{1}$ Division of Surgical Oncology, National Cancer Centre Singapore, Singapore, Singapore. ${ }^{2}$ SingHealth Duke-NUS Breast Centre, Singapore General Hospital, Singapore, Singapore. ${ }^{3}$ Integrated Genomics Platform, National Cancer Centre Singapore, Singapore, Singapore. ${ }^{4}$ Laboratory of Cancer Epigenome, National Cancer Centre Singapore, Singapore, Singapore. ${ }^{5}$ Integrated Biostatistics and Bioinformatics Program, Duke-NUS Medical School, Singapore, Singapore. ${ }^{6}$ Duke-NUS Medical School, Singapore, Singapore.

${ }^{7}$ Department of Anatomical Pathology, Singapore General Hospital, Singapore, Singapore. ${ }^{8}$ Division of Pathology, Singapore General Hospital, Singapore, Singapore.

Received: 10 May 2019 Accepted: 23 September 2019 Published online: 23 October 2019

\section{References}

1. Eble JN, Tavassoli FA, Devilee P, Organization WH, Cancer IAfRo: pathology and genetics of Tumours of the breast and female genital organs: IARC press; 2003.

2. Geisler DP, Boyle MJ, Malnar KF, McGee JM, Nolen MC, Fortner SM, Broughan TA. Phyllodes tumors of the breast: a review of 32 cases. Am Surg. 2000;66(4):360-6.

3. Bernstein L, Deapen D, Ross RK. The descriptive epidemiology of malignant cystosarcoma phyllodes tumors of the breast. Cancer. 1993;71(10):3020-4.

4. Tan PH, Jayabaskar T, Chuah KL, Lee HY, Tan Y, Hilmy M, Hung H, Selvarajan S, Bay BH. Phyllodes tumors of the breast: the role of pathologic parameters. Am J Clin Pathol. 2005;123(4):529-40.

5. Komenaka IK, El-Tamer M, Pile-Spellman E, Hibshoosh H. Core needle biopsy as a diagnostic tool to differentiate phyllodes tumor from fibroadenoma. Arch Surg. 2003;138(9):987-90.

6. Gradishar WJ, Anderson BO, Balassanian R, Blair SL, Burstein HJ, Cyr A, Elias AD, Farrar WB, Forero A, Giordano SH, et al. Breast Cancer, version 4.2017, NCCN clinical practice guidelines in oncology. J Natl Compr Cancer Netw. 2018;16(3):310-20

7. Salvadori B, Cusumano F, Del Bo R, Delledonne V, Grassi M, Rovini D, Saccozzi R, Andreola S, Clemente C. Surgical treatment of phyllodes tumors of the breast. Cancer. 1989;63(12):2532-6.

8. Bilous M. Breast core needle biopsy: issues and controversies. Mod Pathol. 2010;23(Suppl 2):S36-45.

9. Giri D. Recurrent challenges in the evaluation of fibroepithelial lesions. Arch Pathol Lab Med. 2009;133(5):713-21.

10. Lawton TJ, Acs G, Argani P, Farshid G, Gilcrease M, Goldstein N, Koerner F, Rowe JJ, Sanders M, Shah SS, et al. Interobserver variability by pathologists in the distinction between cellular fibroadenomas and phyllodes tumors. Int J Surg Pathol. 2014;22(8):695-8. 
11. Bandyopadhyay S, Barak S, Hayek K, Thomas S, Saeed H, Beydoun R, Shi D, Arabi H, Ruterbusch J, Cote M, et al. Can problematic fibroepithelial lesions be accurately classified on core needle biopsies? Hum Pathol. 2016;47(1):38-44.

12. Tan WJ, Cima I, Choudhury Y, Wei X, Lim JC, Thike AA, Tan MH, Tan PH. A five-gene reverse transcription-PCR assay for pre-operative classification of breast fibroepithelial lesions. Breast Cancer Res. 2016;18(1):31.

13. Lim WK, Ong CK, Tan J, Thike AA, Ng CC, Rajasegaran V, Myint SS, Nagarajan $\mathrm{S}$, Nasir ND, McPherson JR, et al. Exome sequencing identifies highly recurrent MED12 somatic mutations in breast fibroadenoma. Nat Genet 2014;46(8):877-80.

14. Ng CC, Tan J, Ong CK, Lim WK, Rajasegaran V, Nasir ND, Lim JC, Thike AA, Salahuddin SA, lqbal J, et al. MED12 is frequently mutated in breast phyllodes tumours: a study of 112 cases. J Clin Pathol. 2015;68(9):685-91.

15. Yoshida M, Sekine S, Ogawa R, Yoshida H, Maeshima A, Kanai Y, Kinoshita T, Ochiai A. Frequent MED12 mutations in phyllodes tumours of the breast. $\mathrm{Br}$ J Cancer. 2015;112(10):1703-8.

16. Tan J, Ong CK, Lim WK, Ng CC, Thike AA, Ng LM, Rajasegaran V, Myint SS, Nagarajan S, Thangaraju S, et al. Genomic landscapes of breast fibroepithelial tumors. Nat Genet. 2015;47(11):1341-5.

17. Yoshida M, Ogawa R, Yoshida H, Maeshima A, Kanai Y, Kinoshita T, Hiraoka $\mathrm{N}$, Sekine S. TERT promoter mutations are frequent and show association with MED12 mutations in phyllodes tumors of the breast. Br J Cancer. 2015; 113(8):1244-8

18. Piscuoglio S, Ng CK, Murray M, Burke KA, Edelweiss M, Geyer FC, Macedo GS, Inagaki A, Papanastasiou AD, Martelotto LG, et al. Massively parallel sequencing of phyllodes tumours of the breast reveals actionable mutations, and TERT promoter hotspot mutations and TERT gene amplification as likely drivers of progression. J Pathol. 2016;238(4):508-18.

19. Cimino-Mathews A, Hicks JL, Sharma R, Vang R, Illei PB, De Marzo A, Emens $L A$, Argani P. A subset of malignant phyllodes tumors harbors alterations in the Rb/p16 pathway. Hum Pathol. 2013;44(11):2494-500.

20. Feakins RM, Mulcahy HE, Nickols CD, Wells CA. p53 expression in phyllodes tumours is associated with histological features of malignancy but does not predict outcome. Histopathology. 1999;35(2):162-9.

21. Kleer CG, Giordano TJ, Braun T, Oberman HA. Pathologic, immunohistochemical, and molecular features of benign and malignant phyllodes tumors of the breast. Mod Pathol. 2001;14(3):185-90.

22. FibroPhyllo ${ }^{\mathrm{TM}}$ Tissue Test. Make the Right Cut. https://www.lucencedx.com/ category/media-release/. 2018.

23. Koh VCY, Ng CCY, Bay BH, Teh BT, Tan PH. The utility of a targeted gene mutation panel in refining the diagnosis of breast phyllodes tumours. Pathology. 2019.

24. Yeong J, Thike AA, Young Ng CC, Md Nasir ND, Loh K, Teh BT, Tan PH. A genetic mutation panel for differentiating malignant phyllodes tumour from metaplastic breast carcinoma. Pathology. 2017:49(7):786-9.

25. Lakhani SR. WHO classification of Tumours of the breast. International Agency for Research on Cancer. 2012.

26. Thorvaldsdóttir H, Robinson JT, Mesirov JP. Integrative genomics viewer (IGV): high-performance genomics data visualization and exploration. Brief Bioinform. 2013;14(2):178-92.

27. Ihemelandu C, Fernandez S, Sugarbaker PH. A prognostic model for predicting overall survival in patients with peritoneal surface malignancy of an Appendiceal origin treated with Cytoreductive surgery and Hyperthermic Intraperitoneal chemotherapy. Ann Surg Oncol. 2017;24(8):2266-72.

28. Ludyga N, Grunwald B, Azimzadeh O, Englert S, Hofler H, Tapio S, Aubele M. Nucleic acids from long-term preserved FFPE tissues are suitable for downstream analyses. Virchows Arch. 2012;460(2):131-40.

29. Ademà V, Torres E, Solé F, Serrano S, Bellosillo B. Paraffin treasures: do they last forever? Biopreserv Biobank. 2014;12(4):281-3.

30. Guyard A, Boyez A, Pujals A, Robe C. Tran Van Nhieu J, Allory Y, Moroch J, Georges O, Fournet JC, Zafrani ES et al: DNA degrades during storage in formalin-fixed and paraffin-embedded tissue blocks. Virchows Arch. 2017; 471(4):491-500.

31. Do H, Dobrovic A. Sequence artifacts in DNA from formalin-fixed tissues: causes and strategies for minimization. Clin Chem. 2015;61(1):64-71.

32. Cani AK, Hovelson DH, McDaniel AS, Sadis S, Haller MJ, Yadati V, Amin AM, Bratley J, Bandla S, Williams PD, et al. Next-gen sequencing exposes frequent MED12 mutations and actionable therapeutic targets in Phyllodes tumors. Mol Cancer Res. 2015;13(4):613-9.

33. Nagasawa S, Maeda I, Fukuda T, Wu W, Hayami R, Kojima Y, Tsugawa K, Ohta T. MED12 exon 2 mutations in phyllodes tumors of the breast. Cancer Med. 2015:4(7):1117-21.
34. Piscuoglio S, Murray M, Fusco N, Marchiò C, Loo FL, Martelotto LG, Schultheis AM, Akram M, Weigelt B, Brogi E, et al. MED12 somatic mutations in fibroadenomas and phyllodes tumours of the breast. Histopathology. 2015:67(5):719-29.

35. Laé M, Gardrat S, Rondeau S, Richardot C, Caly M, Chemlali W, Vacher S, Couturier J, Mariani O, Terrier P, et al. MED12 mutations in breast phyllodes tumors: evidence of temporal tumoral heterogeneity and identification of associated critical signaling pathways. Oncotarget. 2016;7(51):84428-38.

36. Lien HC, Huang CS, Yang YW, Jeng YM. Mutational analysis of MED12 exon 2 in a spectrum of fibroepithelial tumours of the breast: implications for pathogenesis and histogenesis. Histopathology. 2016;68(3):433-41.

37. Pareja F, Geyer FC, Kumar R, Selenica P, Piscuoglio S, Ng CKY, Burke KA, Edelweiss M, Murray MP, Brogi E, et al. Phyllodes tumors with and without fibroadenoma-like areas display distinct genomic features and may evolve through distinct pathways. NPJ Breast Cancer. 2017;3:40.

38. Marusyk A, Polyak K. Tumor heterogeneity: causes and consequences. Biochim Biophys Acta. 2010;1805(1):105-17.

39. Key Demographic Trends. http://www.singstatgovsg/publications/ publications_and_papers/cop2010/census_2010_advance_census_release/ key_demographic_trendspdf. 2010.

\section{Publisher's Note}

Springer Nature remains neutral with regard to jurisdictional claims in published maps and institutional affiliations.
Ready to submit your research? Choose BMC and benefit from:

- fast, convenient online submission

- thorough peer review by experienced researchers in your field

- rapid publication on acceptance

- support for research data, including large and complex data types

- gold Open Access which fosters wider collaboration and increased citations

- maximum visibility for your research: over $100 \mathrm{M}$ website views per year

At BMC, research is always in progress.

Learn more biomedcentral.com/submissions 